\title{
Point de vue des patients concernant la prise en charge anesthésique
}

\section{[Patient's perspective with regard to anesthesia services]}

\author{
C. Baillard MD, J.F. Lamberto MD, C. Martinez MD, P. Le Toumelin MD, J.L.Fournier MD, M. Cupa MD, \\ C.M. Samama MD PhD
}

Objectif : L'anesthésie s'exerce volontiers en équipe, faisant intervenir pour un même patient, plusieurs praticiens au cours de son séjour hospitalier. L'objectif de ce travail était de recueillir l'opinion des patients sur ce sujet.

Eléments cliniques : Nous avons interrogé 912 patients consécutifs (âge: $51 \pm 16$ ans; sexe: $H 58 \%$ ) à la fin de la consultation d'anesthésie, sur leur préférence éventuelle pour l'anesthésiologiste du bloc opératoire. Cette question était posée par l'infirmière et en l'absence du médecin consultant afin d'éviter une réponse "induite ou polie" en faveur de celui-ci. Avant la fin de l'hospitalisation, le sentiment des patients concernant le fait de ne pas avoir été pris en charge par le même anesthésiologiste, lorsque c'était le cas, était réévalué. Finalement, l'identité des praticiens ayant participé à leur prise en charge était demandée.

A la fin de la consultation, 34\% des patients exprimaient un choix pour l'anesthésiologiste du bloc opératoire, celui-ci allant toujours vers le médecin consultant, les femmes marquant plus fréquemment leur préférence. Interrogés avant leur sortie de l'hôpital, 80\% des patients n'avaient pas été endormis par l'anesthésiologiste consultant et parmi ceux-ci, $4 \%$ le regrettaient. Le nom du chirurgien était le plus fréquemment mémorisé (60\%) et celui de l'anesthésiologiste du bloc opératoire le moins bien connu (5\%).

Conclusion : La prise en charge anesthésique comprenant différents anesthésiologistes n'apparaît pas comme une préoccupation majeure pour les patients interrogés. Des efforts doivent être réalisés pour améliorer notre identification auprès des patients.

Purpose: Whereas patients most often select their surgeon, they don't usually select their anesthesiologist. Further, anesthesia frequently involves different physicians at different stages perioperatively. This inability to choose and the multiplicity of interveners may reduce patient satisfaction. Our study examined patients' willingness to choose their anesthesiologist for the operation, as well as their opinion on the way anesthesia is practiced presently.
Clinical features: Nine hundred and twelve patients (mean age 51 $\pm 16 \mathrm{yr}, 58 \%$ males) were requested, immediately after the preoperative visit, to choose their anesthesiologist for the operation. The request was formuled by a nurse, in the absence of the anesthesiologist, in order to avoid a courteous response. After surgery, prior to leaving the hospital, patients were invited to give their opinion on the anesthesia care received and to name the anesthesiologist(s) and surgeon involved.

Results: Women chose an anesthesiologist more frequently than men, but only $34 \%$ of patients overall elected to do so. Eighty percent of patients were cared for by an anesthesiologist other than the one seen at the preoperative visit. Four percent of patients regretted the change. Patients recalled the surgeon's name more frequently (60\%) than the attending anesthesiologist's (4\%).

Conclusion: In France, the preoperative visit is required by law and must precede the operation by at least $48 \mathrm{hr}$. Despite this preoperative interview, patients were unwilling to choose the anesthesiologist responsible for surgery, did not object to multiple interveners and seldom remembered their anesthesiologist's name. Efforts to improve the image of the profession are required.

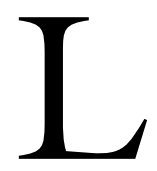

'ANESTHÉSIE s'exerce volontiers avec de multiples intervenants (consultation, bloc opératoire et suivi post- opératoire), rendant difficile la personnalisation de la relation médecin-patient. Cette situation peut expliquer, au moins en partie, la difficulté des patients à identifier les anesthésiologistes. ${ }^{1,2}$ Il est licite de penser que ce problème, purement organisationnel pourrait nuire à la relation médecin-patient. Une pratique commune et consensuelle (dossier unique, procédure validée, compte rendu de consultation) permet néanmoins d'offrir au patient une prise en charge et un suivi adaptés à défaut d'une réelle personnalisation. La volonté

Département d'anesthésie, Hôpital Avicenne, Bobigny, France.

Adresser la correspondance ḋ: Dr C. Baillard, Département d'anesthésie, Hôpital Avicenne, 125 route de Stalingrad, 93009 Bobigny

cedex, France. Téléphone: 331489555 91; Télécopieur: 331489555 89; E-mail: christophe.baillard@avc.ap-hop-paris.fr.

Accepted for publication November 9, 2000.

Revision accepted February 12, 2001. 
TABLEAU Influence des variables liées au patient sur la volonté de choisir l'anesthésiologiste du bloc opératoire

\begin{tabular}{llll}
\hline Variable & Odds ratio & IC 95\% & $P$ \\
\hline Sexe féminin & 2,26 & $1,37-3,7$ & 0,004 \\
Spécialité chirurgicale & 1,04 & $0,92-1,17$ & 0,55 \\
Âge & 1,01 & $0,99-1,03$ & 0,1 \\
Profession & 0,84 & $0,66-1,08$ & 0,18 \\
\hline
\end{tabular}

Régression logistique multivariée, $n=912$.

des patients dans ce domaine mérite d'être clarifiée car plus de la moitié d'entre eux apparaissent insensibles à l'annonce d'un changement d'anesthésiologiste entre la consultation et le bloc opératoire. ${ }^{1}$ Des facteurs sociaux et/ou démographiques peuvent également influencer leur choix. ${ }^{2}$

Ce travail avait pour objectif de recueillir l'opinion des patients sur ce sujet en précisant: leur volonté de choisir le médecin anesthésiologiste, leur ressenti concernant leur prise en charge ainsi que la mémorisation de l'identité des intervenants.

\section{Méthode}

À la fin de la consultation d'anesthésie, les patients adressés pour chirurgie réglée (non urgente), ont été interrogés afin de préciser s'ils désiraient choisir un anesthésiologiste pour le bloc opératoire. La question posée par l'infirmière de la consultation, en l'absence du médecin, était libellée comme suit: "Désirez vous choisir un médecin anesthésiologiste pour être endormi au bloc opératoire?". Les caractéristiques socio-démographiques des patients étaient relevées: âge, sexe, profession (sans profession, chômage, employé, cadre, étudiant, retraité), ainsi que la chirurgie pour laquelle les patients étaient adressés. Les anesthésiologistes consultants étaient au nombre de quatorze (quatre femmes, dix hommes). La prise en charge des patients n'a pas été modifiée au cours de la période étudiée.

Avant la sortie de l'hôpital, lors de la dernière visite post-opératoire réalisée en salle, nous avons proposé aux patients de répondre aux questions suivantes: (i) connaissez-vous le nom du chirurgien qui a réalisé votre intervention?; (ii) connaissez-vous le nom de l'anesthésiologiste vu en consultation?; (iii) connaissez-vous le nom de l'anesthésiologiste qui a réalisé l'anesthésie au bloc opératoire?; (iv) connaissez-vous le nom d'un anesthésiologiste vous ayant pris en charge? Finalement, le sentiment des patients concernant le fait de ne pas avoir été pris en charge par le même anesthésiologiste, lorsque c'était le cas, était réévalué.
Statistiques: test de ${ }^{2}$ ou test exact de Fischer pour les données qualitatives et test $t$ de Student pour les données quantitatives avec calcul des odds ratio. Les analyses multidimensionnelles ont utilisé une régression logistique descendante et ascendante pas à pas utilisant les variables suivantes: âge, sexe, profession et spécialité chirurgicale. Une valeur de $P<0,05$ était considérée comme significative. Toutes les données ont été traitées avec le logiciel SAS version 6.12 (SAS institute, Cary, NC, USA).

\section{Résultats}

Sur une période de deux mois, 912 patients consécutifs (hommes: 55\%) adressés en consultation d'anesthésie ont été interrogés. L'âge moyen était de $51 \pm 17$ ans (médiane 52, extrêmes: 18-86 ans). Les patients étaient adressés pour chirurgie orthopédique $(46 \%)$, vasculaire $(17 \%)$, thoracique $(16 \%)$, otorhinolaryngologique $(10 \%)$, digestive, endoscopique et ophtalmologique (11\%). En ce qui concerne la profession exercée, les patients étaient employés (49\%), cadres $(19 \%)$, retraités $(16 \%)$, étudiants $(5 \%)$ ou sans profession (11\%).

Aucun patient n'a refusé de répondre à notre enquête et $34 \%$ d'entre eux ont exprimé un choix pour un anesthésiologiste. Ce choix, lorsqu'il était exprimé, était toujours en faveur de l'anesthésiologiste consultant. Parmi les caractéristiques des patients, seul le sexe intervenait significativement (Tableau), les femmes marquant plus fréquemment leur préférence (odds ratio 2,26, $P<0,05$ ). L'identité ou le sexe de l'anesthésiologiste consultant n'intervenaient pas dans la réponse du patient.

Alors que $80 \%$ des patients n'avaient pas été endormis par l'anesthésiologiste vu en consultation, seulement $4 \%$ d'entre eux le regrettaient.

Le nom du chirurgien était le plus fréquemment mémorisé (60\%) et celui de l'anesthésiologiste du bloc opératoire le moins bien connu (5\%). Dans 17\% des cas les patients se souvenaient du nom de l'anesthésiologiste de la consultation préopératoire. Les patients ayant été endormis par l'anesthésiologiste de la consultation ne mémorisaient pas mieux son identité.

\section{Discussion}

La majorité des patients n'exprime pas de préférence pour l'anesthésiologiste du bloc opératoire. Dans l'étude de Shevde et al., seulement $21 \%$ des patients désiraient choisir leur anesthésiologiste ${ }^{2}$ et un travail français récent retrouve également les mêmes tendances. ${ }^{1}$ Ce résultat est, à première vue, surprenant puisqu'il ne s'inscrit pas dans une démarche qualité, souhaitée par les patients, valorisant la personnalisation de la relation patient-médecin. ${ }^{3,4}$ 
Dans le travail de Shevde et $a l^{2}$ l'absence de choix reposait essentiellement sur le fait que les patients ne connaissaient pas les (autres) anesthésiologistes. Dans notre étude, toutes les réponses positives allant exclusivement vers l'anesthésiologiste consultant renforcent cette hypothèse. D'autre part, il a été montré que les patients peuvent ignorer que l'anesthésiologiste consultant est un médecin. ${ }^{5-7}$

Toutefois, interrogés avant la sortie de l'hôpital, les patients ne regrettent pas que leur prise en charge ait impliqué plusieurs anesthésiologistes. Ce résultat semble démontrer que les patients sont peu sensibles à la notion d'interlocuteur unique. Il est également possible que la notion d'équipe soit perçue favorablement par l'enrichissement des compétences qu'elle procure, à condition que celle-ci ait été expliquée. La prise en charge anesthésique comprenant différents anesthésiologistes n'apparaît donc pas comme une préoccupation majeure pour le patient.

Interrogées sur des questions relatives à l'anesthésiologie, les femmes se montrent globalement plus intéressées. ${ }^{2,8,9}$ Dans notre étude, elles expriment plus fréquemment la volonté de choisir le médecin anesthésiologiste du bloc opératoire. Les différences de comportements sociaux liées au sexe peuvent expliquer qu'un même problème s'exprime différemment, l'homme étant moins enclin à exprimer ses craintes. ${ }^{2,5}$

L'anesthésiologiste travaille souvent dans l'anonymat. On comprend aisément que l'identité de l'anesthésiologiste du bloc opératoire soit oubliée par la situation de stress, la prémédication et les effets amnésiants des hypnotiques. Néanmoins, l'anesthésiologiste de la consultation est constamment moins bien identifié que le chirurgien. La signification de cet anonymat, déjà relevée dans les travaux de Vedrinne et Zvara mérite d'être clarifiée. ${ }^{1,10}$ Une des hypothèses retenue est que le patient choisit son chirurgien, alors que l'anesthésiologiste lui est le plus souvent imposé.

Le nom de l'anesthésiologiste est oublié dès la sortie de la consultation ${ }^{1}$ ce qui tend à prouver que la mémorisation n'est pas en cause mais plutôt les moyens permettant l'identification. La multiplicité des intervenants complique encore un peu plus la situation mais son rôle n'est probablement pas très important puisque les patients ayant été endormis par l'anesthésiologiste de la consultation ne mémorisent pas mieux son identité. La répétition des visites postopératoires n'améliore pas non plus l'identification des anesthésiologistes. ${ }^{10}$ Enfin, bien qu'il n'y ait pas de relation entre l'identification de l'anesthésiologiste et la reconnaissance de son travail par le patient, l'anonymat pourrait être favorisé par la connaissance imparfaite de son rôle.

\section{Conclusion}

La prise en charge anesthésique comprenant de multiples anesthésiologistes ne semble pas être une préoccupation majeure pour la population étudiée. Les femmes expriment plus volontiers le souhait de choisir le médecin anesthésiologiste. L'identité des anesthésiologistes est très mal connue des patients. Des efforts doivent être consentis pour mieux s'identifier auprès des patients.

\section{Références}

1 Védrinne C, Laroux MC, Blanc P, Durand PG, George $M$, Lehot JJ. Consultation d'anesthésie de chirurgie cardiovasculaire et thoracique. Enquête de satisfaction des patients et des médecins. Ann Fr Anesth Réanim 1999; 18: 834-42.

2 Shevde K, Panagopoulos G A survey of 800 patients'knowledge, attitudes, and concerns regarding anesthesia. Anesth Analg 1991; 73: 190-8.

3 Penon C, Ecoffey C Evaluation par les patients de la qualité de la prise en charge anesthésique. Ann Fr Anesth Réanim 1995; 14: 374-5.

4 Hume MA, Kennedy B, Asbury AJ. Patient knowledge of anaesthesia and peri-operative care. Anaesthesia 1994; 49: 715-8.

5 Klafta JM, Roizen MF. Current understanding of patients' attitudes toward and preparation for anesthesia: a review. Anesth Analg 1996; 83: 1314-21.

6 Keep PJ, Jenkins JR. As others see us : the patient's view of the anaesthetist. Anaesthesia 1978; 33: 43-5.

7 Simini B. Anaesthetist: the wrong name for the right doctor. Lancet 2000; 355: 1892.

8 van Wijk MGF, Smalhout B. A postoperative analysis of the patient's view of anaesthesia in a Netherlands' teaching hospital. Anaesthesia 1990; 45: 679-82.

9 Lonsdale M, Hutchison GL. Patient's desire for information about anaesthesia. Scottish and Canadian attitudes. Anaesthesia 1991; 46: 410-2.

10 Zvara DA, Nelson JM, Brooker RF et al. The importance of the postoperative anesthetic visit: do repeated visits improve patient satisfaction or physician recognition? Anesth Analg 1996; 83: 793-7. 